\title{
Latest results concerning short range correlations obtained in the dp elastic and dp breakup processes at Nuclotron, JINR
}

Marian Janek $^{1 \star}$, Vladimir P. Ladygin ${ }^{2}$, Alexander V. Averyanov ${ }^{2}$, Eugene V. Chernykh $^{2}$, Dan D. Enache ${ }^{3}$, Yuri V. Gurchin ${ }^{2}$, Alexandr Yu. Isupov ${ }^{2}$, Julia-Tatiana Karachuk ${ }^{3}$, Anatoly N. Khrenov ${ }^{2}$, Dimitry O. Krivenkov ${ }^{2}$, Pavel K. Kurilkin ${ }^{2}$, Nadezhda B. Ladygina $^{2}$, Alexei N. Livanov ${ }^{2}$, Olena Mezhenska ${ }^{4}$, Semen M. Piyadin ${ }^{2}$, Sergei G. Reznikov ${ }^{2}$, Yaroslav T. Skhomenko ${ }^{2,5}$, Arkady A. Terekhin ${ }^{2}$, Alexei V. Tishevsky ${ }^{2}$ and Tomohiro Uesaka ${ }^{6}$

1 Physics Department, University of Zilina, Univerzitna 1, 01001 Zilina, Slovakia, 2 Veksler and Baldin Laboratory of High Energies, Joint Institute for Nuclear Research, Joliot-Curie 6, 141980 Dubna, Moscow region, Russia

3 Advanced Research Institute for Electrical Engineering, Splaiul Unirii 313, Bucharest, Romania

4 Institute of Physics, University of P. J. Shafarik, Park Angelinum 9, 04001 Kosice, Slovakia

5 Belgorod State University, Pobedy 85, 308015 Belgorod, Russia

6 Nishima Center for Accelerator-based Science, RIKEN, 351-0198 Wako, Japan

$\star$ janek.marian@gmail.com

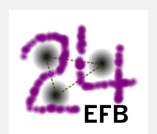

Proceedings for the 24th edition of European Few Body Conference, Surrey, UK, 2-6 September 2019

doi:10.21468/SciPostPhysProc.3

\begin{abstract}
Deuteron spin structure program is aimed on extraction of two and three nucleon forces information, including their spin dependent parts, from dp elastic and dp breakup processes investigated at intermediate energies. The dp elastic data were obtained at Internal Target Station of Nuclotron (JINR) in the energy range $400-1800 \mathrm{MeV}$ using polarized deuteron beam. Strong sensitivity to the short range spin structure of the isoscalar nucleon-nucleon correlations is observed in deuteron analyzing powers. Preliminary results of the the cross section for the dp breakup reaction have been obtained at $400 \mathrm{MeV}$ of deuteron energy.
\end{abstract}

(c) (1) Copyright M. Janek et al.

This work is licensed under the Creative Commons

Attribution 4.0 International License.

Published by the SciPost Foundation.
Received 14-10-2019

Accepted 05-11-2019

Published 24-02-2020

doi:10.21468/SciPostPhysProc.3.011

\section{Introduction}

There are many evidences that realistic nucleon-nucleon (NN) potentials such as CD-Bonn [1], AV18 [2] and Nijm I, II and 93 [3] does not describe the NN data above $~ 350 \mathrm{MeV}$. Binding energies of three [4] and four nucleon systems can not be described using only NN potentials, however theoretical models in which three nucleon forces (3NFs) are included as two pion 
exchange, e.g. Urbana IX [5] and Tucson-Melbourne [6] give us reasonable agreement. The cross section of reactions with few nucleons involving is in general better described in unpolarized case than in the polarized one. In the vicinity of the Sagara discrepancy the currently known $3 \mathrm{NFs}$ contribute by up to $30 \%$ to the $d p$ elastic scattering cross section at intermediate energies [7].

The $d p$ breakup reaction at deuteron energy of $270 \mathrm{MeV}$ was investigated at RIKEN [8] and IUCF [9]. It was found that vector analyzing power $A_{y}$ can be described using NN forces only, but other polarization observables need 3NFs to describe the data. Inclusion of 3NFs improve the description of a part of the data but breaks other. Relativistic effects for the nd elastic scattering cross section at $70 \mathrm{MeV}$ and $250 \mathrm{MeV}$ were investigated in [24]. It was found that relativistic effects contribution is located mainly at backward angles, but their contribution is not large enough to fill discrepancy between experimental data and theory, even in the case when standard three nucleon forces are used.

The main goal of Deuteron Spin Structure program (DSS) is to obtain information about two and three nucleon forces, including their spin dependent parts. For this purpose, $d p$ elastic scattering and $d p$ breakup reaction are investigated in the energy ranges from $300 \mathrm{MeV}-2000$ $\mathrm{MeV}$ and $300 \mathrm{MeV}-500 \mathrm{MeV}$ of deuteron energy.

\section{Dp elastic scattering at $400 \mathrm{MeV}-2000 \mathrm{MeV}$}

Polarimetry at Internal Target Station (ITS) [11] is using $d p$ elastic scattering at large scattering angles $\left(\theta_{c m} \geq 60^{\circ}\right)$ at $270 \mathrm{MeV}$ [12], where precise data on analyzing powers [13] exist. Method gives us determination of deuteron polarization better than $2 \%$. Polarimeter consists of a spherical hull in which up to six targets can be placed. There are 39 scintillation counters placed at the top, bottom, left and right with respect to the deuteron beam, downstream to the ITS of Nuclotron. Scattered deuterons and recoil protons are detected in the coincidence in angular range from $65^{\circ}-135^{\circ}$. Developed multichannel high voltage system power supply system can supply power to 70 Hamamatsu photomultipliers. Polarization measurements were performed with new control and data acquisition system [14]. Developed new polarized ion source (SPI) [15] provided polarization of deuteron beam whose ideal values are: $P_{Z}, P_{Z Z}=(0,0),(-1 / 3,-1)$ and $(-1 / 3,+1)$. The measured values of beam polarization were from $\sim 65 \%$ to $\sim 75 \%$ of ideal values. Observed beam polarizations were reasonably stable and demonstrate good reproducibility after interruptions. The selection of $d p$ elastic events are based on correlations of energy losses in scintillators for the proton and deuteron and by difference in theirs time of flight information. Additionally, in order to suppress background cut on interaction point between beam and target was applied, also.

The angular dependence of the cross section at deuteron energy of $1400 \mathrm{MeV}$ is shown in Figure. 1. Curves are predictions based on relativistic-multiple scattering model, e.g. [16] when one nucleon exchange and single scattering term (dashed curve), additional double scattering term (dotted curve) and $\Delta$ isobar (solid curve) are included. Nuclotron data are represented by closed symbols, world data at slightly different energies are shown by open symbols. One can see large contribution which comes from double scattering term. It describes the data up to $70^{\circ} . \Delta$ isobar contribution comes at larger angles above $80^{\circ}$ and rises with angle. However the data are described in this angular range only qualitatively.

The angular dependence of the vector $A_{y}$, tensor $A_{y y}$ and $A_{x x}$ at deuteron energy of 400 $\mathrm{MeV}$ is shown in Figure.2. Curves are predictions based on relativistic-multiple scattering model when one nucleon exchange and single scattering term (dashed curve) and additional double scattering term (solid curve) are taken into account. Nuclotron data are represented by closed symbols, open ones are world data [17], [18] and [19]. The contribution which comes 


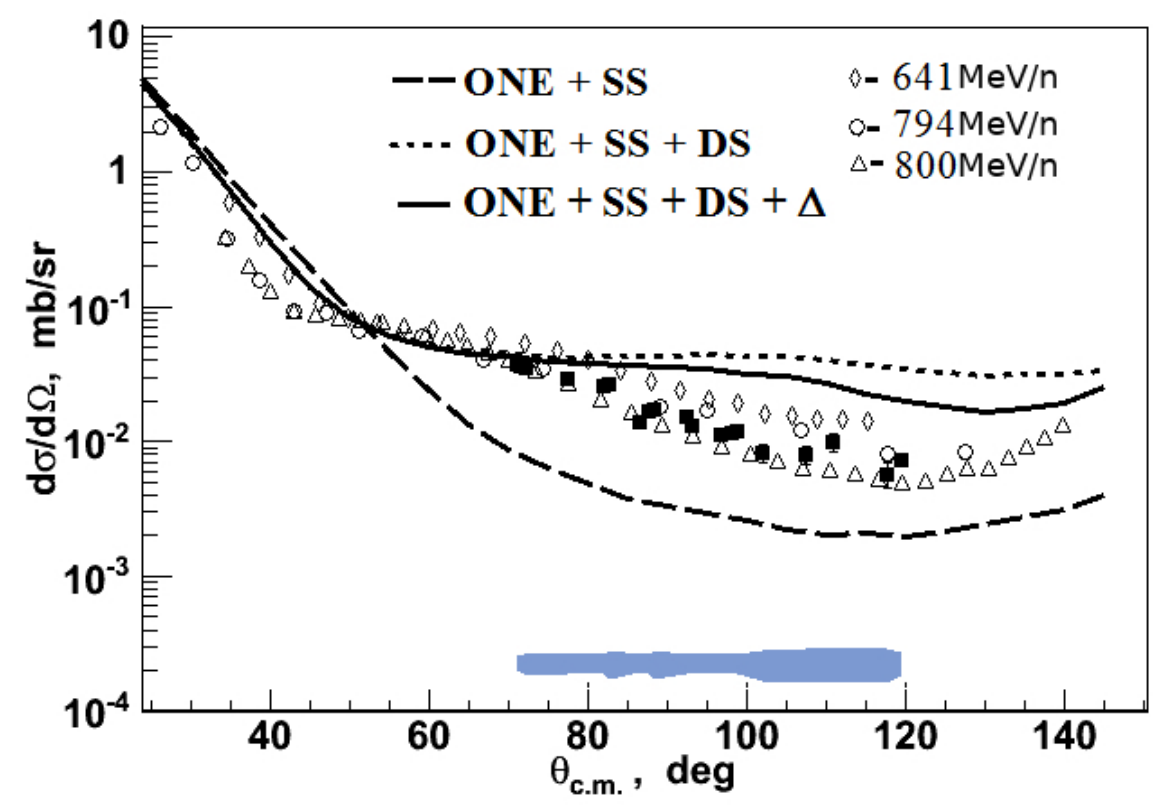

Figure 1: Angular dependence of the cross section at deuteron energy of $1400 \mathrm{MeV}$. Curves are predictions based on relativistic-multiple scattering model when one nucleon exchange and single scattering term (dashed curve), additional double scattering term (dotted curve) and $\Delta$ isobar (solid curve) are included. Nuclotron data are represented by closed symbols, world data at slightly different energies are shown by open symbols.

from double scattering term is rather small at this energy and generally does not improve the description of the data. Vector $A_{y}$ and tensor $A_{y y}$ analyzing powers are described up to the $70^{\circ}$. Tensor $A_{x x}$ analyzing powers is not described by the model. The reason of the deviation can be neglecting of three nucleon short range correlations.

Angular dependence of the vector $A_{y}$, tensor $A_{y y}$ and $A_{x x}$ at deuteron energy of $700 \mathrm{MeV}$ is shown in Figure.3. Curves are predictions based on relativistic-multiple scattering model when one nucleon exchange and single scattering term (dashed curve), additional double scattering term (dotted curve) and $\Delta$ isobar (solid curve) are taken into account. One can see large contribution which comes from double scattering term and small contribution from $\Delta$ isobar located in very backward angles. Very good description is obtained in case of tensor analyzing power $A_{y y}$.

The Angular dependence of the vector $A_{y}$, tensor $A_{y y}$ and $A_{x x}$ at deuteron energy of 1000 $\mathrm{MeV}$ is shown in Figure.4. Curves are the same as for the case of $700 \mathrm{MeV}$. One can see large contribution which comes from double scattering term and moderate to large contribution which comes from $\Delta$ isobar, specially at angles larger than $140^{\circ}$. Only part of the $A_{y}$ data up to $70^{\circ}$ is described. Possible reason is that spin structure of the NN interactions and deuteron is missing in relativistic multiple scattering model [16].

\section{Dp breakup process at $300 \mathrm{MeV}-500 \mathrm{MeV}$}

$D p$ breakup data have been accumulated using polarized and unpolarized deuteron beam at $300 \mathrm{MeV}, 400 \mathrm{MeV}$ and $500 \mathrm{MeV}$. Analyzing powers $i T_{11}$ and $T_{20}$ are investigated at deuteron energy of $400 \mathrm{MeV}$. There are two important settings in which unpolarized data are collected. 


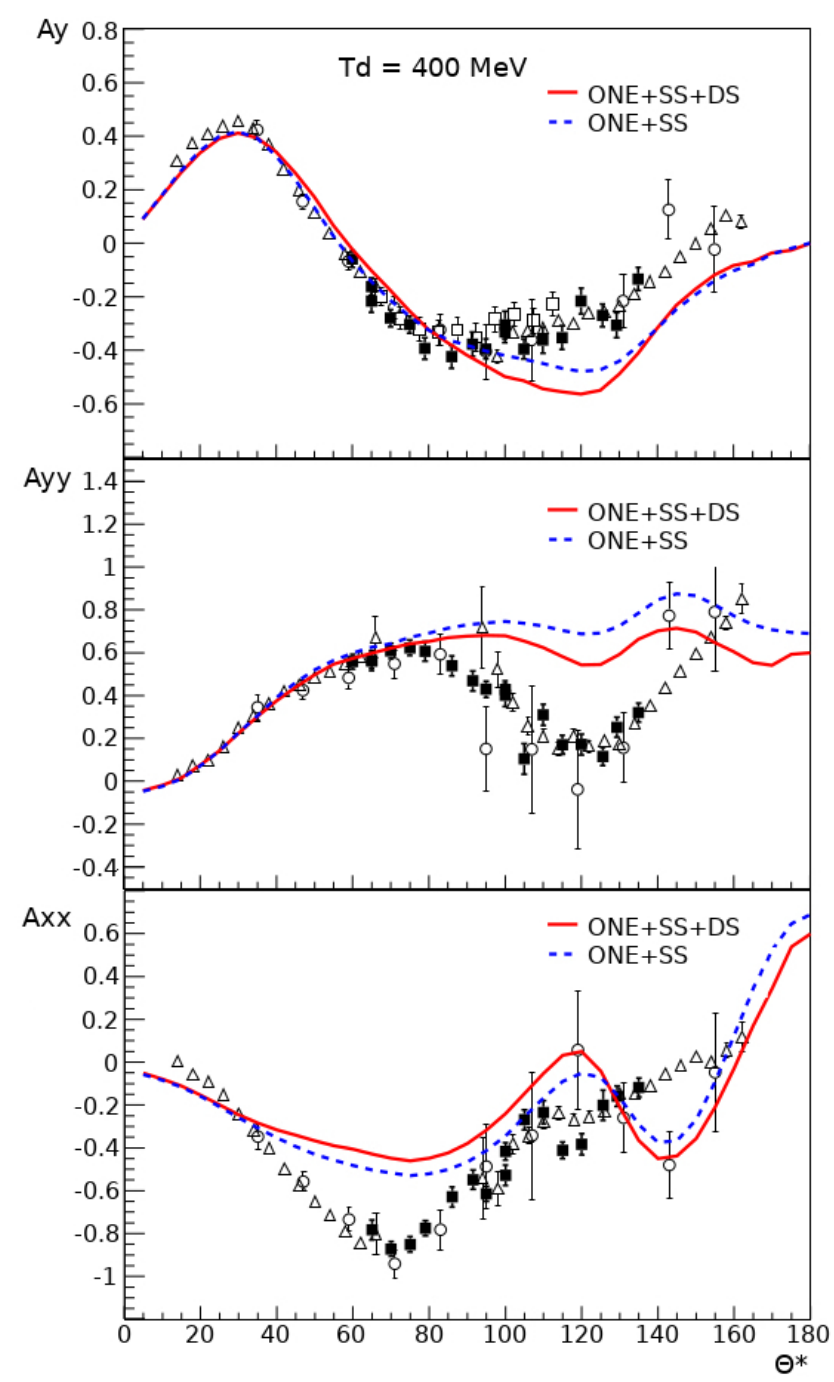

Figure 2: Angular dependence of the vector $A_{y}$, tensor $A_{y y}$ and $A_{x x}$ at deuteron energy of $400 \mathrm{MeV}$. Curves are predictions based on relativistic-multiple scattering model when one nucleon exchange and single scattering term (dashed curve) and additional double scattering term (solid curve) are taken into account. Nuclotron data are represented by closed symbols, open symbols represent world data.

First settings could give us possibility to investigate two and three nucleon forces, second one could be suitable in searching for relativistic effects. In the first setting, the detectors are placed in configurations in which momentum vectors of outgoing particles have equal momenta in the center of mass system and are separated by $120^{\circ}$. Detectors were placed in various configurations in so called intermediate star. In the second setting, one arm is fixed and second scans angular range.

Analyzing powers $i T_{11}$ and $T_{20}$ were obtained at deuteron energy of $400 \mathrm{MeV}$ at ITS of Nuclotron. Values of polarization of deuteron beam were obtained using polarimetry method described in section 2. Polyethylene and Carbon targets are enclosed in a spherical hull of ITS. Up to six various targets can be placed inside of ITS. Details of the $\Delta E-E$ detector construction can be found in [20]. $i T_{11}$ analyzing power at $72.3^{\circ}$ and $76.5^{\circ}$ in centre of mass system was measured under $p p$ quasielastic conditions. Obtained values at $72.3^{\circ}$ and $76.5^{\circ}$ are $0.10 \pm 0.02$ and $0.11 \pm 0.06$, respectively. Results are in good agreement with the world $p p$ - quasielastic scattering data within obtained experimental errors. Values of the vector $i T_{11}$ and tensor $T_{20}$ 


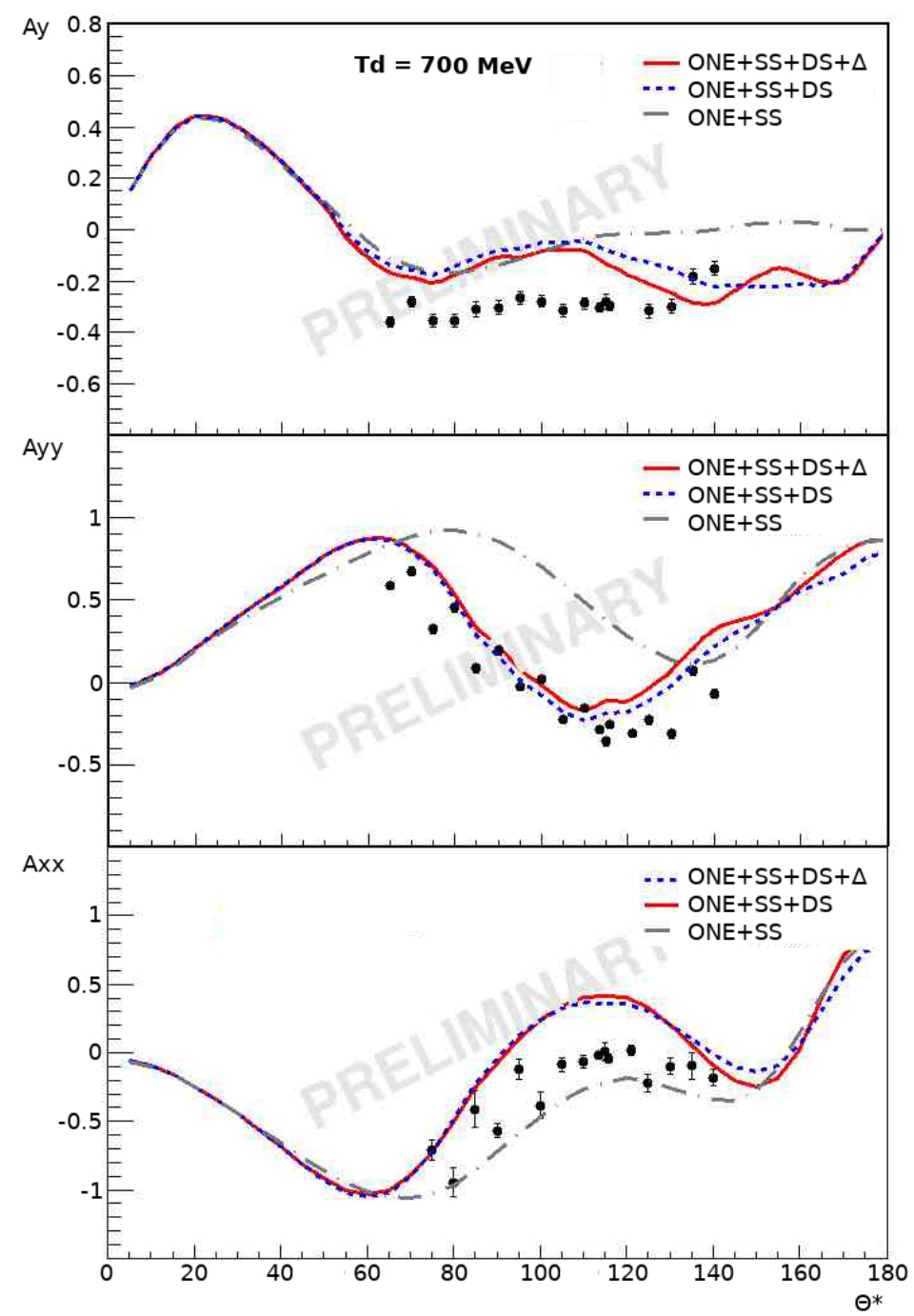

Figure 3: Angular dependence of the vector $A_{y}$, tensor $A_{y y}$ and $A_{x x}$ at deuteron energy of $700 \mathrm{MeV}$. Curves are predictions based on relativistic-multiple scattering model when one nucleon exchange and single scattering term (dashed curve), additional double scattering term (dotted curve) and $\Delta$ isobar (solid curve) are taken into account.

analyzing powers at polar angles of $34.8^{\circ}$ and $36.8^{\circ}$ and difference between azimuthal angles of $135^{\circ}$ are $0.47 \pm 0.10$ and $0.02 \pm 0.20$ [21]. Beam luminosity needs to be increase at least of one order in order to substantially decrease errors of analyzing powers.

$D p$ breakup reaction using unpolarized deuteron beam has been investigated at $300 \mathrm{MeV}$, $400 \mathrm{MeV}$ and $500 \mathrm{MeV}$ of deuteron energy. $\Delta \mathrm{E}$-E correlation of energy losses of charged particles and prediction which comes from GEANT4 simulation is shown in Figure.5. One can see good agreement between reconstructed data and simulation. Reconstructed energy spectra obtained on Polyethylene and Carbon in case of deuteron energy of $400 \mathrm{MeV}$ are shown at the bottom of Figure.5, where left and right panels are related to left and right arms of detector, open and closed histograms represent data obtained on Polyethylene and Carbon targets. Reasonable agreement between experiment and GEANT4 simulation was obtained [22]. One can see clear peak at the value of $100 \mathrm{MeV}$ in both arms which comes from $p p$ - quasielastic events. The $p p$ - quasielastic reaction at $90^{\circ}$ in centre of mass and at the three energies of 300 $\mathrm{MeV}, 400 \mathrm{MeV}$ and $500 \mathrm{MeV}$ was used to obtain calibration constants. The stability of ampli- 


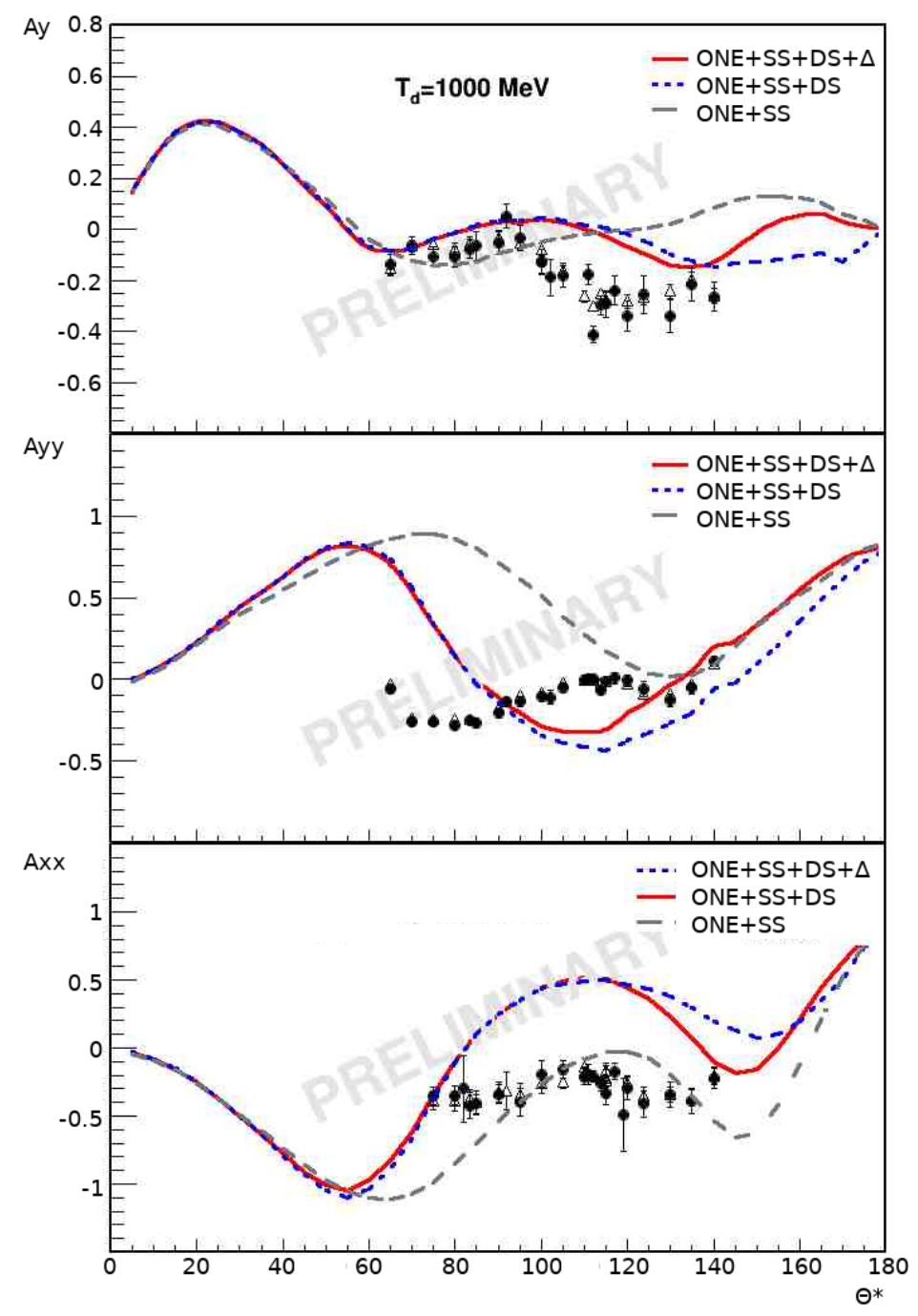

Figure 4: Angular dependence of the vector $A_{y}$, tensor $A_{y y}$ and $A_{x x}$ at deuteron energy of $1000 \mathrm{MeV}$. Curves are predictions based on relativistic-multiple scattering model when one nucleon exchange and single scattering term (dashed curve), additional double scattering term (dotted curve) and $\Delta$ isobar (solid curve) are taken into account.

tude of all photomultiplier tubes (PMTs) were monitored during all data acquisition. Detailed description of LED system of PMTs can be found in [20]. Calibration procedure is described in [23].

Relativistic effects have been found to be important in $n d$ breakup reaction at $200 \mathrm{MeV}$ in special kinematics in which one arm is fixed and second scans angular interval [24]. It was found specific pattern in differential cross section as a function of scattering angle. Relativistic contribution varies from no contribution to very large one. We performed the measurement of $d p$ breakup reaction in inverse kinematics at deuteron energy of $400 \mathrm{MeV}$. First arm is fixed at angle of $43^{\circ}$ and second one scans the angular range. Second arm takes the following angular values: $27^{\circ}, 31^{\circ}, 35^{\circ}$ and $39^{\circ}$.

Preliminary results of the five fold differential cross section of $d p$ breakup reaction investigated at $400 \mathrm{MeV}$ for the case of detector arms placed at the angles of $31^{\circ}$ and $43^{\circ}, 35^{\circ}$ and $43^{\circ}, 39^{\circ}$ and $43^{\circ}$ are shown in Figure.6. Only statistical errors are shown. $S-$ variable repre- 

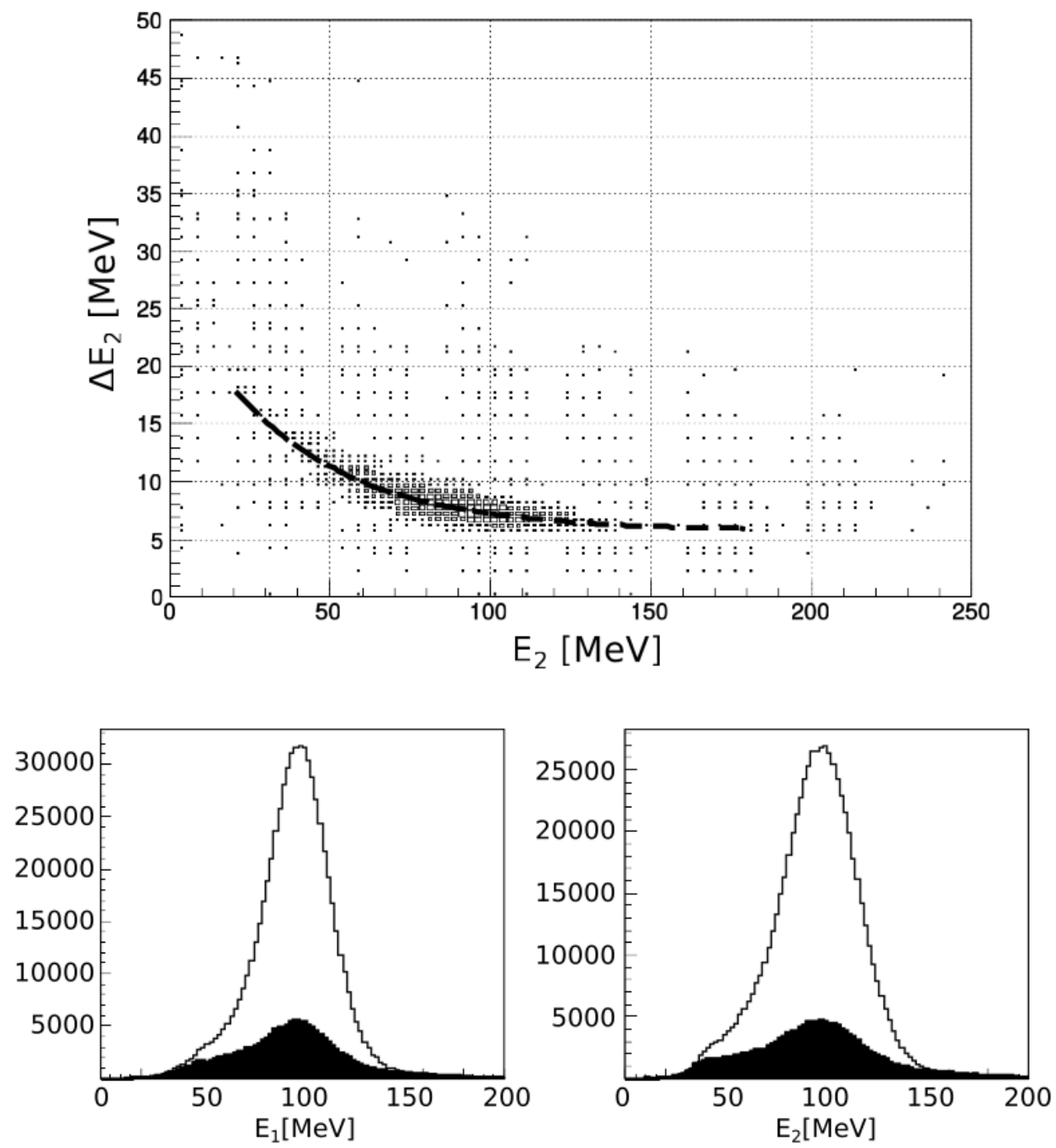

Figure 5: Top: $\triangle \mathrm{E}-\mathrm{E}$ correlation of energy losses of charged particles (mainly protons) and prediction which comes from GEANT4 simulation (dashed line). Bottom: Energy spectra obtained on Polyethylene and Carbon in case of deuteron energy of $400 \mathrm{MeV}$. Left and right panels are related to left and right arms of detector. Open and closed histograms represent data obtained on Polyethylene and Carbon targets.

sents arc-length of kinematic curve in the plot of two registered protons. $S$ - variable interval is cut due to detector acceptance. Preliminary data show some structures at the vicinity of $\approx 100 \mathrm{MeV}$ and $\approx 260 \mathrm{MeV}$.

\section{Conclusion}

Cross section of $d p$ elastic scattering and predictions of relativistic-multiple scattering model at $1400 \mathrm{MeV}$ is presented. Good agreement is observed up to $70^{\circ}$. Angular dependence of the vector $A_{y}$, tensor $A_{y y}$ and $A_{x x}$ at deuteron energy of 400,700 and $1000 \mathrm{MeV}$ is presented along with the predictions based on relativistic multiple scattering model. Large contribution which comes from double scattering term is observed, but it does not improve the description of analyzing powers in all cases, rather at small angles. Moderate contribution which comes 


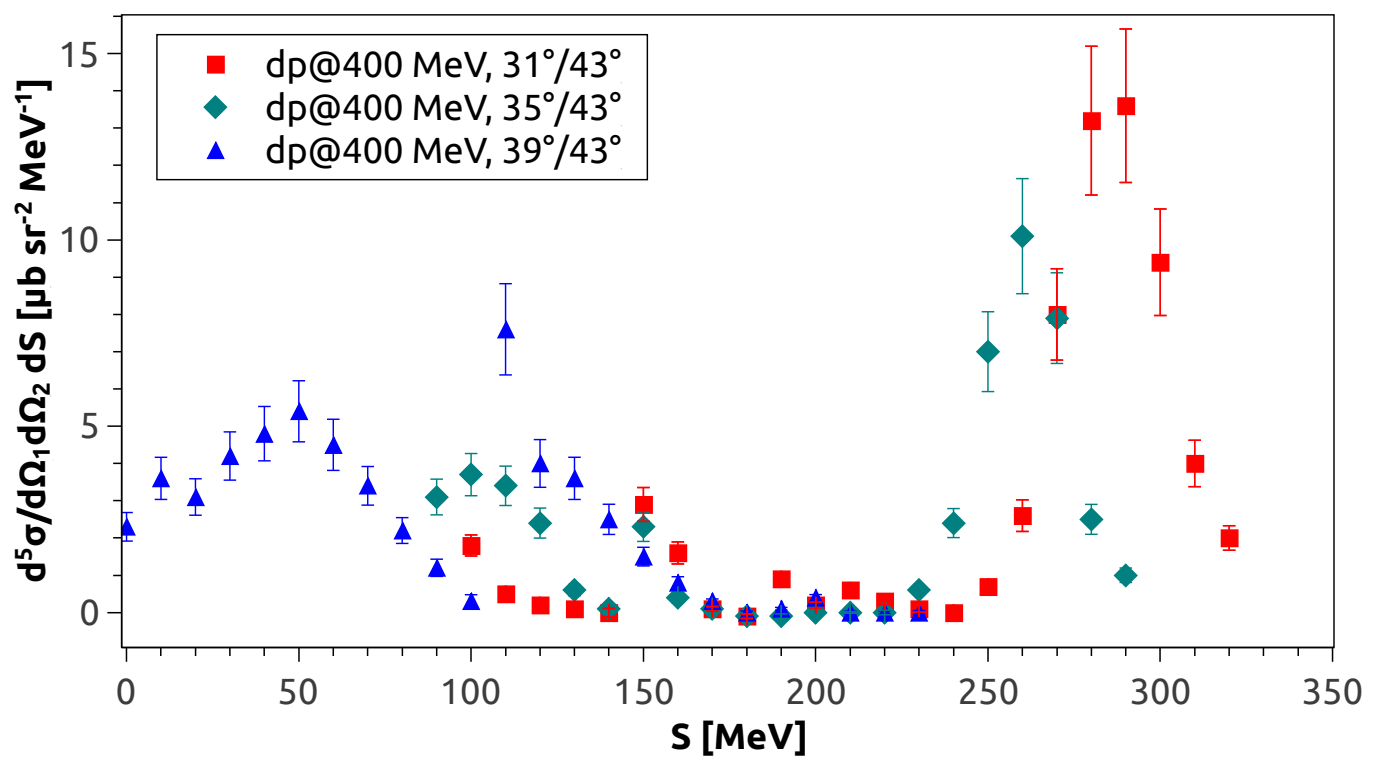

Figure 6: Preliminary results of the five fold differential cross section of $d p$ breakup reaction investigated at $400 \mathrm{MeV}$ for the case of detector arms placed at the angles of $31^{\circ}$ and $43^{\circ}$ (red symbols), $35^{\circ}$ and $43^{\circ}$ (green symbols), $39^{\circ}$ and $43^{\circ}$ (blue symbols), respectively. Only statistical errors are shown.

from $\Delta$ isobar is found at higher energies. $\Delta$ isobar contribution improves the qualitative description of the cross section at $1400 \mathrm{MeV}$.

Preliminary results of the five fold differential cross section of $d p$ breakup reaction investigated at $400 \mathrm{MeV}$ for the case of detector arms placed at the angles of $31^{\circ}$ and $43^{\circ}, 35^{\circ}$ and $43^{\circ}, 39^{\circ}$ and $43^{\circ}$ are presented. One can see some structures in kinematic $S$ - curve at the vicinity of $\approx 100 \mathrm{MeV}$ and $\approx 260 \mathrm{MeV}$. The next step is to obtain results at other angles to investigate observed structure in recent data.

\section{Acknowledgements}

The authors thank the Nuclotron staff for providing good conditions of the experiment and for the tune of the polarized ion source SPI [15].

Funding information The work has been supported in part by the Ministry of Education, Science, Research and Sport of the Slovak Republic VEGA Grant No. 1/0113/18 (M.J.), by the RFBR under grant No.19-02-00079a (V. P. L.) and by JINR- Slovak Republic cooperation programs (M. J. and V. P .L.) in 2019.

\section{References}

[1] S. A. Coon and W. Glöckle, Two-pion-exchange three-nucleon potential: Partial wave analysis in momentum space, Phys. Rev. C 23, 1790 (1981), doi:10.1103/PhysRevC.23.1790.

[2] S. A. Coon, M. D. Scadron, P. C. McNamee, B. R. Barrett, D. W. E. Blatt and B. H. J. McKellar, The two-pion-exchange three-nucleon potential and nuclear matter, Nucl. Phys. 
A 317, 242 (1979), doi:10.1016/0375-9474(79)90462-7.

[3] V. G. J. Stoks, R. A. M. Klomp, C. P. F. Terheggen and J. J. de Swart, Construction of high-quality NN potential models, Phys. Rev. C 49, 2950 (1994), doi:10.1103/PhysRevC.49.2950.

[4] H. R. Setze et al., Cross-section measurements of the space-star configuration in N-D breakup at 13.0 MeV, AIP Conf. Proc. 334, 463 (1995), doi:10.1063/1.48131.

[5] G. Rauprich, S. Lemaître, P. Niessen, K. R. Nyga, R. Reckenfelderbaumer, L. Sydow, H. Paetz Gen. Schieck, H. Witała and W. Glöckle, Study of the kinematically complete breakup reaction ${ }^{2} H(p, p p) n$ at $E_{p}=3 \mathrm{MeV}$ with polarized protons, Nucl. Phys. A 535, 313 (1991), doi:10.1016/0375-9474(91)90451-B.

[6] H. Patberg et al., Deuteron breakup reaction ${ }^{2} H(\vec{p}, p p) n$ induced by polarized protons at $E_{p}=19.0 \mathrm{MeV}$, Phys. Rev. C 53, 1497 (1996), doi:10.1103/PhysRevC.53.1497.

[7] N. Sakamoto et al., Measurement of the vector and tensor analyzing powers for the d-p elastic scattering at $E_{d}=270 \mathrm{MeV}$, Phys. Lett. B 367, 60 (1996), doi:10.1016/03702693(95)01398-9.

[8] K. Sekiguchi et al., Three-nucleon force effects in the ${ }^{1} H(\vec{d}, \vec{p} p) n$ reaction at 135 $\mathrm{MeV} /$ nucleon, Phys. Rev. C 79, 054008 (2009), doi:10.1103/PhysRevC.79.054008.

[9] H. O. Meyer et al., Axial observables in $\vec{d} \vec{p}$ breakup and the three-nucleon force, Phys. Rev. Lett. 93, 112502 (2004), doi:10.1103/PhysRevLett.93.112502.

[10] H. Witała, J. Golak, R. Skibiński, W. Glöckle, W. N. Polyzou and H. Kamada, Relativistic effects in neutron-deuteron elastic scattering and breakup, Few-Body Syst. 49, 61 (2010), doi:10.1007/s00601-010-0098-4.

[11] A. I. Malakhov et al., Potentialities of the internal target station at the Nuclotron, Nucl. Instr. Meth. in Phys. Res. Sec. A 440, 320 (2000), doi:10.1016/S0168-9002(99)009663.

[12] P. K. Kurilkin et al., The $270 \mathrm{MeV}$ deuteron beam polarimeter at the Nuclotron Internal Target Station, Nucl. Instr. Meth. in Phys. Res. Sec. A 642, 45 (2011), doi:10.1016/j.nima.2011.03.054.

[13] K. Sekiguchi et al., Complete set of precise deuteron analyzing powers at intermediate energies: Comparison with modern nuclear force predictions, Phys. Rev. C 65, 034003 (2002), doi:10.1103/PhysRevC.65.034003.

[14] A. Yu. Isupov, V. A. Krasnov, V. P. Ladygin, S. M. Piyadin and S. G. Reznikov, The Nuclotron internal target control and data acquisition system, Nucl. Instr. Meth. in Phys. Res. Sec. A 698, 127 (2013), doi:10.1016/j.nima.2012.09.057.

[15] V. V. Fimushkin, A. D. Kovalenko, L. V. Kutuzova, Y. V. Prokofichev, B. Shutov, A. S. Belov, V. N. Zubets and A. V. Turbabin, Development of polarized ion source for the JINR accelerator complex, J. Phys.: Conf. Ser. 678, 012058 (2016), doi:10.1088/17426596/678/1/012058.

[16] N. B. Ladygina, Delta excitation in deuteron-proton elastic scattering, Eur. Phys. J. A 52 , 199 (2016), doi:10.1140/epja/i2016-16199-5. 
[17] M. Garcon et al., Measurements of vector and tensor analysing powers for 191 and $395 \mathrm{MeV}$ deuteron scattering, Nucl. Phys. A 458, 287 (1986), doi:10.1016/0375-9474(86)903581.

[18] R. V. Cadman et al., Evidence for a three-nucleon-force effect in proton-deuteron elastic scattering, Phys. Rev. Lett. 86, 967 (2001), doi:10.1103/PhysRevLett.86.967.

[19] B. v. Przewoski et al., Analyzing powers and spin correlation coefficients for $p+d$ elastic scattering at 135 and $200 \mathrm{MeV}$, Phys. Rev. C 74, 064003 (2006), doi:10.1103/PhysRevC.74.064003.

[20] S. M. Piyadin et al. [DSS collaboration], $\Delta E-E$ detector for proton registration in nonmesonic deuteron breakup at the Nuclotron internal target, Phys. Part. Nuclei Lett. 8, 107 (2011), doi:10.1134/S1547477111020105.

[21] M. Janek et al., Analyzing powers $i T_{11}$ and $T_{20}$ of $d p \rightarrow$ ppn reaction at $400 \mathrm{MeV}$ investigated at Nuclotron Communications: Scientific letters of the University of Zilina, 3, 62 (2017).

[22] M. Janek, B. Trpišová, S. M. Piyadin and V. P. Ladygin, GEANT4 simulation of dp nonmesonic breakup reaction at 300 and $500 \mathrm{MeV}$, Phys. Part. Nuclei Lett. 11, 552 (2014), doi:10.1134/S1547477114040219.

[23] M. Janek et al., Calibration procedure of the $\Delta E-E$ detectors for dp breakup investigation at nuclotron, Phys. Part. Nuclei Lett. 15, 76 (2018), doi:10.1134/S1547477118010090.

[24] H. Witała, J. Golak, R. Skibiński, W. Glöckle, W. N. Polyzou and H. Kamada Relativistic effects in neutron-deuteron elastic scattering and breakup, Few-Body Syst. 49, 61 (2011), doi:10.1007/s00601-010-0098-4. 